\title{
Chemisorptive electron emission versus sticking probability
}

\author{
Artur Böttcher* \\ Fritz-Haber-Institut der Max-Planck-Gesellschaft, Faradayweg 4-6, 14195 Berlin, Germany \\ Horst Niehus \\ Institut für Physik der Humboldt-Universität, Invalidenstrasse 110, 10115 Berlin, Germany
}

(Received 28 November 2000; published 29 June 2001)

\begin{abstract}
The chemisorption of $\mathrm{N}_{2} \mathrm{O}$ on thin Cs films has been studied by monitoring the time evolution of the sticking probability as well as the kinetics of the low-energy electron emission. By combining the data sets, two time domains become distinguishable: the initial chemisorption stage is characterized by a high sticking probability $(0.1<S<1)$ and by a rather weak low-energy electron emission. The opposite is the case within the late stage where the chemisorption saturates, a very intense electron emission is accompanied by the negligibly low sticking probability of less than 0.01. Such evident anticoincidence between the exoemission and the chemisorption excludes the model of surface harpooning as the elementary process responsible for the electron emission in the late chemisorption stage. A long-term emission decay has also been observed after turning off the flux of chemisorbing molecules. A model is proposed that attributes both, the late chemisorptive and the nonchemisorptive electron emission to the relaxation of a narrow state originated from an oxygen vacancy in the Cs oxide layer terminating the surface. The presence of such a state has been confirmed by the metastable de-excitation spectroscopy [MDS, $\left.\mathrm{He}^{*}\left(2^{1} S\right)\right]$.
\end{abstract}

DOI: 10.1103/PhysRevB.64.045407

PACS number(s): 73.20.-r, 79.75.+g, 82.65.+r

\section{INTRODUCTION}

Low-energy electron emission has been observed during the chemisorption of electronegative molecules on metallic surfaces. ${ }^{1-4}$ It is well established that the observed electron emission manifests a nonadiabatic path of the chemisorptive event, i.e., it reflects a kind of surface excitation created when the empty affinity state, lowest unoccupied molecular orbital, (LUMO) of the chemisorbing molecule dives deeply below the Fermi level as an unoccupied state (Ref. 4 and references therein). The necessary condition for a possible appearance of such a short living hole is a considerable quenching of the resonant electron transfer at the Fermi level. This very efficient resonant process drastically reduces the probability that the hole can dive deeply enough in respect to the Fermi level for inducing a detectable electron emission, $E^{*}>E_{F}\left(E^{*}\right.$ denotes the hole energy in respect to the vacuum level). The obvious energy requirement, $E^{*}$ $>2 \Phi$, where $\Phi$ represents the local work function, is automatically fulfilled for metal surfaces marked by low work function values as well as for strongly electronegative molecules $\left(\mathrm{F}_{2}, \mathrm{Cl}_{2}, \mathrm{NO}_{2}\right.$, etc). In this case the empty affinity level is located close to the Fermi level, $E_{a} \approx E_{F}$, even at long surface-molecule distances. Consequently the affinity state of an impinging molecule crosses the Fermi region far away from the surface, without becoming charged and survives as a hole at an energy of $E^{*}>2 \Phi$, where the surfacemolecule electron transfer releases an Auger cascade, which finally leads to an intense electron emission.

The molecule-surface interaction takes place on the time scale shorter than $10^{-12} \mathrm{~s}$. This time interval is estimated for thermal molecules that pass through the surface-near region defined by the harpooning distance and the location of the image plane, in the range of $1-10 \AA .{ }^{1}$ Thus, the molecular dynamics of the chemisorbing molecules might directly be measured by using a time-resolved spectroscopy with a resolution of $10^{-15} \mathrm{~s}$ and a spatial resolution of better than $1 \AA$. Such extreme requirements cannot be fulfilled yet. An alternative way would be to work with pulsed molecular beams. Unfortunately, the time resolution in this case is strongly limited by the achievable chopper frequency, which for standard mechanical rotors is longer than one microsecond. Consequently, the only way left to reconstruct the dynamics of chemisorbing molecules was to perform steady-state experiments ${ }^{1-7}$ with various properly chosen surfaces (lowwork-function metals, structured surfaces, etc.) or with molecules differing by their physical properties (affinity level, dissociation energy, etc.). Some details of the molecular dynamics were derived by fitting the steady-state results to theoretical models, which related the survival probability of a LUMO to striking properties of the impinging molecules. ${ }^{8-11}$

The chemisorptive electron emission induced by moderately electronegative molecules (e.g., $\mathrm{O}_{2}$ or $\mathrm{N}_{2} \mathrm{O}$ ) has been attributed to the dissociation event that creates atomic unoccupied states. $^{12-15}$ This chemisorptive scenario has mainly been supported by two experimental findings. It has been shown that the intensity of exoelectron emission strongly depends on the primary velocity of the impinging molecules ${ }^{15,16}$ as well as on their vibronic excitation state. ${ }^{17,18}$ Thus, when the exoemission is induced by surface harpooning, a simple proportionality between the sticking coefficient for impinging molecules and the resulting emission intensity is expected. This relationship however, is not supported by proper experiments yet.

In the following we report on the possibility of closing this gap by measuring the correlation between the intensity of the exoelectron emission and the probability for dissociative chemisorption by the example of $\mathrm{N}_{2} \mathrm{O}$ molecules chemisorbing on $\mathrm{Cs}$ films deposited on $\mathrm{Ru}$ (0001). This particular choice is motivated by several aspects: A detailed knowledge 
of the electronic and geometric structure of Cs layers deposited on $\mathrm{Ru}$ (0001) has recently been achieved. ${ }^{19-21}$ Hence, it is possible to relate some elementary steps of the postulated nonadiabatic path to specific properties of the $\mathrm{Cs} / \mathrm{Ru}$ target more precisely. On the practical point of view, the nitrous oxides as pollutants are efficient ozone consumers in the Earth's atmosphere. Thus, a pronounced effort has recently been undertaken for applying new alkali-metal supported catalysts, which allows us to reduce the $\mathrm{N}_{2} \mathrm{O}$ emission considerably. ${ }^{22,23}$

\section{EXPERIMENT}

The experiments were performed in an ultrahigh vacuum chamber at a base pressure of less than $10^{-10} \mathrm{mbar}$. The (UHV) chamber is equipped with standard facilities for crystal cleaning and characterization [low-energy electron diffraction (LEED), Auger, ultraviolet photoemission spectroscopy (UPS), and quadrapole mass spectrometer (QMS)]. It is connected with a molecular-beam apparatus, which provided a nearly monoenergetic molecular flux by supersonic gas expansion (nozzle diameter $10 \mu \mathrm{m}$ ). Under such conditions the molecular flux reached a level of $10^{13}$ molecule $/ \mathrm{cm}^{2} \mathrm{~s}$ with the beam cross section covering more than $90 \%$ of the sample surface. The $\mathrm{N}_{2} \mathrm{O}$ beam was directed under $22.5^{\circ}$ to the surface normal and the scattering molecular fluxes were monitored by a mass spectrometer (extranuclear) mounted along the specular direction. All experiments were performed with thermal molecules, i.e., with a nozzle kept at room temperature. The molecular flux has been determined by measuring the oxygen load reached by exposing the clean $\mathrm{Ru}$ surface to the molecular beam and comparing this value with the one obtained by performing the gas dosage once more by flooding the chamber up to several partial pressures in the range of $10^{-8}-10^{-7}$ mbar. The resulting oxygen coverage has been determined by taking the oxygen thermal desorption spectra and relating the integral of the TD traces to the spectrum, which represents the saturated layer of chemisorbed oxygen ( 1 Monolayer $(\mathrm{ML})=1.58$ $\times 10^{15} \mathrm{~cm}^{-2}$ ). The time evolution of the sticking coefficient has been determined according to the King-Wells method, by monitoring the flux of scattered molecules. ${ }^{25}$

The chemisorptive exoelectron emission has been analyzed by the means of a hemispherical electron energy spectrometer working online during exposing the Cs films to the molecular beam. The entrance slit of the energy analyzer was located in front of the sample with its center at the surface normal. This way the normal component of the entire emission is monitored within an acceptance angle of about $\pm 6^{\circ}$. The spectrometer was designed to provide high electron transmittance at an energy resolution better than $0.4 \mathrm{eV}$. This allowed us to achieve high quality electron energy profiles (4 $\mathrm{eV}, 40$ points, $S / N>10$ ) via an accumulation of the single scans within time intervals shorter than $3 \mathrm{~s}$. The current intensity of the emission has been obtained by integrating these profiles. All experiments with exoelectron emission were performed in the UHV chamber without any sources of visible light. In this way a possible contribution of the photoelectron emission has been excluded.

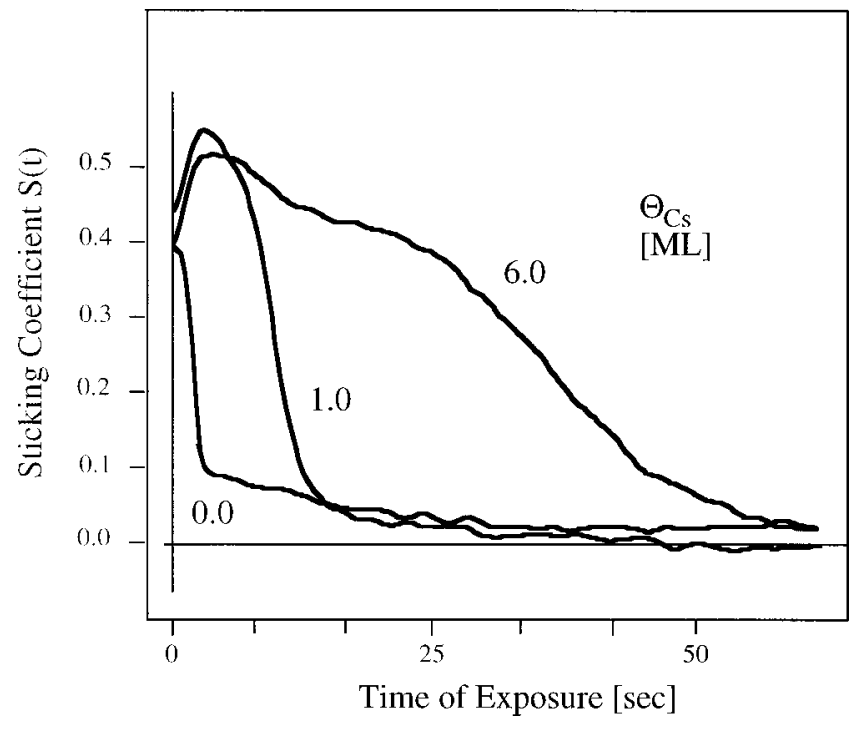

FIG. 1. Time evolution of the sticking coefficient measured by scattering of a $\mathrm{N}_{2} \mathrm{O}$ beam on three Cs layers differing by Cs load deposited, $\Theta_{\mathrm{Cs}}=0,1$ and $6 \mathrm{ML}$. The samples were kept at a constant temperature of $250 \mathrm{~K}$. All experiments were performed at nozzle temperature of $300 \mathrm{~K}$.

The Ru crystal has been cleaned by applying sputtering and annealing cycles according to the procedure described by Madey, Engelhardt, and Menzel. ${ }^{24}$ The achieved surface cleanliness was controlled by applying the UPS as well as the metastable de-excitation spectroscopy ( $\mathrm{He}^{*}$-MDS) and the diffuse He scattering.

Cs films have been deposited by an evaporation from the SAES getter source onto the Ru sample kept at $250 \mathrm{~K}$. The resulting Cs load has been determined by taking thermaldesorption spectra of the deposited Cs atoms and comparing the integral intensity with the one obtained for a saturated Cs monolayer $\left(1 \mathrm{ML}=5.3 \times 10^{14}\right.$ atom $\left./ \mathrm{cm}^{2}\right)$. Cs submonolayers were prepared via thermal desorption of a thick Cs film up to a certain sample temperature $T_{\text {cov }}$, which defines the remaining Cs coverage $\Theta_{\mathrm{Cs}}$. The relationship between $\Theta_{\mathrm{Cs}}$ and $T_{\text {cov }}$ has previously been precisely determined by combining the TDS, Auger, and LEED data. ${ }^{19-21}$

\section{RESULTS AND DISCUSSION}

Figure 1 shows the time evolution of the sticking coefficient $S(t)$, as measured during the exposure of three Cs films $\left(\Theta_{\mathrm{Cs}}=0,1\right.$, and $\left.6 \mathrm{ML}\right)$ to a $\mathrm{N}_{2} \mathrm{O}$ beam of 3 $\times 10^{13}$ molecules $/ \mathrm{cm}^{2} \mathrm{~s}$. The initial sticking probability for $\mathrm{N}_{2} \mathrm{O}$ molecules chemisorbing on a clean substrate $\left(\Theta_{\mathrm{Cs}}\right.$ $=0$ ) reaches a value of 0.4 , which is in agreement with measurements performed by Shi, Lee, and White. ${ }^{26}$ It decreases quickly within the next $5 \mathrm{~s}$, afterwards the decay slows down. After about $30 \mathrm{~s}$ the chemisorption is completed, i.e., the intensity of scattered $\mathrm{N}_{2} \mathrm{O}$ molecules saturates. In the presence of $\mathrm{Cs}$ atoms on $\mathrm{Ru}(0001)$ the initial sticking probability varies only slightly $\left(0.45<S_{\text {init }}<0.5\right)$, however the modifications of the late time evolution $S(t)$ are more pronounced and can be directly related to the increasing Cs load deposited. The most striking difference, a con- 
siderable prolongation of the active stage becomes evident for thicker Cs layers $\left(\Theta_{\mathrm{Cs}}>1\right)$. For example, the sticking probability measured for a 6 ML thick Cs film vanishes only for exposition times longer than $60 \mathrm{~s}$. The integral of the sticking traces is nearly proportional to the thickness of the deposited Cs films. For thick Cs films, the chemisorption kinetics deviates fairly from the predictions based on the langmuir chemisorption scheme applied for two-dimensional (2D) substrates, which of course offer a finite number of adsorption sites. ${ }^{27}$ It becomes intelligible when considering the Cs diffusion through the already created oxide layer as the step limiting the reaction rate. The ability of the partly oxidized Cs films to dissociate impinging molecules is governed by Cs atoms emerging at the topmost surface layer. This process proceeds very efficiently because of an rather low activation barrier for the thermal-diffusion driven transport of Cs atoms of about $0.3 \mathrm{eV}$ as recently found for the oxidation of thick Cs films. ${ }^{3}$ All $S(t)$ traces commonly exhibit an initial increase of the sticking probability in the range of less than $10 \%$ of the initial value. This effect can be explained by the well known fact of a work-function lowering $(\Delta \Phi)$ induced by initial incorporation of oxygen atoms ${ }^{6,26,28,29} \Delta \Phi$ raises the efficiency as well as the distance of the surface harpooning, which more efficiently transfers the impinging molecules into molecular anions and consequently significantly increases the probability for dissociative sticking. ${ }^{1-6}$ The time evolution of the sticking coefficient is very similar to the kinetics of the $N_{2}$ abstraction accompanying the $\mathrm{N}_{2} \mathrm{O}$ chemisorption on Cs films. ${ }^{37}$ This similarity reflects the common origin of the two behaviors, the harpooning-mediated dissociation of the chemisorbing molecules, $\mathrm{N}_{2} \mathrm{O}+[\mathrm{Cs}]_{\text {surf }} \longrightarrow \mathrm{N}_{2}$ (gas) $+\mathrm{O}_{\text {ad }} /[\mathrm{Cs}]_{\text {surf }}$.

Figure 2 shows a correlation between the kinetics of exoelectron emission $I(t)$, and the time evolution of the sticking probability $S(t)$ as measured for three representative Cs layers differing by the Cs load, $0.85,1$, and $6 \mathrm{ML}$, panels $a, b$, and $c$, respectively. For Cs submonolayers [Fig. 2(a)] the exoelectron emission reaches a maximum at the beginning of the exposure, i.e., within the first $3 \mathrm{~s}$ afterwards it disappears gradually. After $15 \mathrm{~s}$ no emission could be observed. This is a common feature for all Cs coverages less than $1 \mathrm{ML}$. It reflects the gradual increasing density of Cs-O bonds created due to the progressing chemisorption. The time evolution of the sticking probability $S(t)$ roughly follows that of the emissive response $I(t)$, i.e., after reaching the maximum it decreases almost simultaneously with the decaying emission. For the saturated 2D layer of adsorbed Cs atoms [Fig. 2(b)], the coincidence between the $S(t)$ and $I(t)$ functions is no longer preserved. In addition to the intense initial emission, a second component of the emission kinetics appears at the late chemisorption stage. The corresponding sticking probability however, proceeds like the one measured for submonolayers, it accompanies only the initial emission component. The intense emission observed at the late chemisorption stage, is accompanied by a drastically decreasing sticking coefficient, which becomes only scarcely distinguishable from the noise.

The relationship between $I(t)$ and $S(t)$ within the late oxidation stage becomes even more pronounced for thick Cs layers [Fig. 2(c)]. Here, the initial emission behaves as the one observed for a monolayer (stage $A$ ) but the late emission kinetics is dominated by a much more intense second maximum at around $60 \mathrm{~s}$ (stage $B$ ). Indeed it coincides with the disappearance of the sticking probability. The clear anticoincidence between the sticking and the exoemission holds for the whole stage $B$.

The exoemission observed at $\mathrm{Cs}$ submonolayers $\left(\Theta_{\mathrm{Cs}}\right.$ $\leqslant 1$ ) has been attributed to the Auger de-excitation induced when the diving molecular affinity state becomes charged via the long-distance resonant surface harpooning. ${ }^{6,17,18}$ Hence, the immediate emission results from an electron transfer event that takes place at the surface-vacuum interface on the time scale shorter than $10^{-12} \mathrm{~s}$. In addition, it has been shown that $\mathrm{N}_{2} \mathrm{O}$ molecules in the excited vibrational state $(h \nu=73 \mathrm{meV})$ are much more exoactive than the groundstate molecules. ${ }^{17,18}$ The coincidence between $S$ and $I$ as found for Cs submonolayers supports the concept of longdistance harpooning as the key step leading to exoemission. The anticoincidence found for thick Cs layers implies, however, that the physical process responsible for the observed electron emission cannot exclusively be related to the surface harpooning as the $\mathrm{N}_{2} \mathrm{O}$-flux conditioned surface-molecule process. Instead, the emissive event has to take place when the $\mathrm{N}_{2} \mathrm{O}$ molecule is already adsorbed on top of the created oxidized layer.

It is generally accepted that the late chemisorption stage $(B)$ starts when the Cs film becomes terminated by an oxide layer. ${ }^{6,30,32}$ UP spectra taken during the oxidation of thick Cs films revealed that the initial stage $A$ is accompanied by a gradually decreasing work function, ranging from $2.1 \mathrm{eV}$ down to about $1 \mathrm{eV} .{ }^{6}$ The minimum value is characteristic for Cs suboxides $\left(\mathrm{CsO}_{7}, \mathrm{Cs}_{3} \mathrm{O}_{11}\right)$ which are composed of dissolved $\mathrm{O}^{--}$ions surrounded by a metallic environment. ${ }^{6,34}$ The beginning second oxidation stage manifests itself by a much more pronounced maximum in the electron emission appearing when the surface work function reaches the minimum value. By continuing the exposure to the $\mathrm{N}_{2} \mathrm{O}$ flux only a slight work-function increase has been observed. Instead, a very slow decay of the emission intensity accompanying the progressing oxidation was observed. Thus, the exoemission kinetics does not depend on the time evolution of the work function, which strongly supports the concept of the delayed, surface mediated emission as the dominating reaction path in the late oxidation stage.

The anticoincidence shown above becomes even more intriguing when monitoring the electron emission after interrupting the $\mathrm{N}_{2} \mathrm{O}$ flux. The exoemission does not disappear entirely, it is continued at a lowered level for several minutes! The left panel in Fig. 3 shows the corresponding emission kinetics. By shutting down the $\mathrm{N}_{2} \mathrm{O}$ beam $(A 1)$ a very fast decay of the total pressure was observed. In order to avoid the possible influence of the residual gas, we restarted the monitoring of the emission only when the pressure in the chamber attained the base level (point $A 2, p<10^{-10} \mathrm{mbar}$ ). Thus, it can entirely be excluded that the emission observed within the time interval $A 2-A 3$ is induced by some chemisorptive processes. Moreover, the energy distributions of the emitted electrons taken at characteristic points $A 1, A 2$, and 

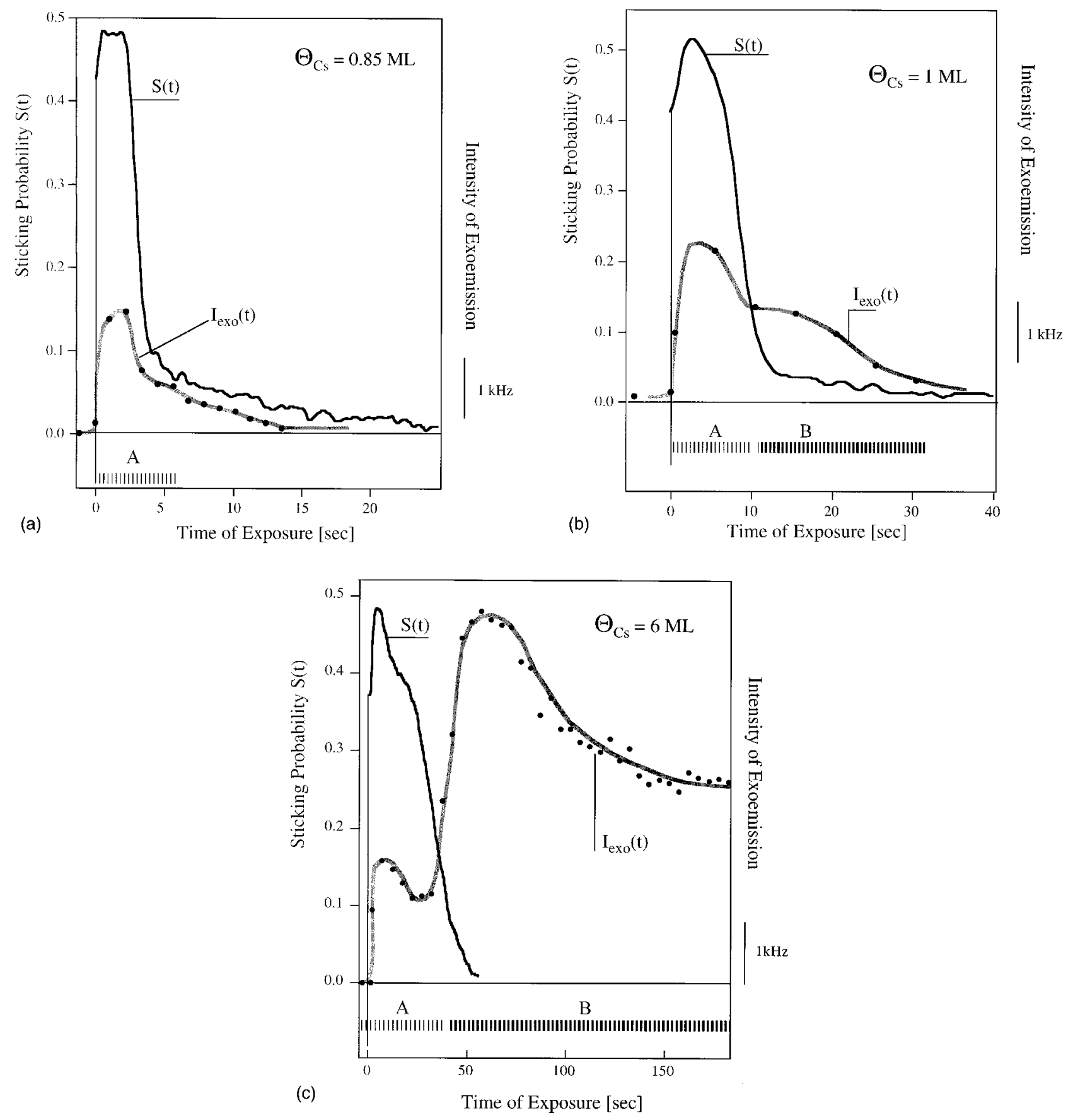

FIG. 2. Kinetics of exoemission in comparison with the time evolution of the sticking probability as monitored for the Cs submonolayer, monolayer, and thick film, (a), (b), and (c) respectively. The same $\mathrm{N}_{2} \mathrm{O}$ flux of $3 \times 10^{13}$ molecule/ $/ \mathrm{cm}^{2} \mathrm{~s}$ has been used for measuring both the sticking coefficient as well as the intensity of exoemission. The latter was measured by integrating the energy distributions of the emitted electrons.

A3, (right panel in Fig. 3) exhibit nearly the same energy location of the maximum intensity as well as a common maximum kinetic energy, $E_{\text {kin, max }}$, at about 2.7-2.9 eV. In addition, all energy profiles exhibit an unusual kind of asymmetry on the low-energy side, which extends over a range broader than $1 \mathrm{eV}$. This cannot be explained by a bad resolution of the detector used here because our energy spectra of the exoemission induced by oxygen chemisorption exhibit a steep flank narrower than $0.4 \mathrm{eV}$. Hence, the location of the maximum intensity is not governed by the current position of the cutting vacuum level and it can be signified as placed about $0.8 \mathrm{eV}$ above the vacuum level. All these characteristic features indicate that the emitted electrons cannot be attributed to the Auger-like excitation of the Cs- $6 s$ electrons of the rather broad conduction band $(\Delta=1.8 \mathrm{eV}$, Ref. 30). This statement is supported by the fact that within the late oxidation stage, the metallic Cs film is buried by a rather thick oxidelike layer, which inhibits the access for impinging molecules. Thus, we might postulate that the emission proceeds via an excitation of a rather localized state, which is located 

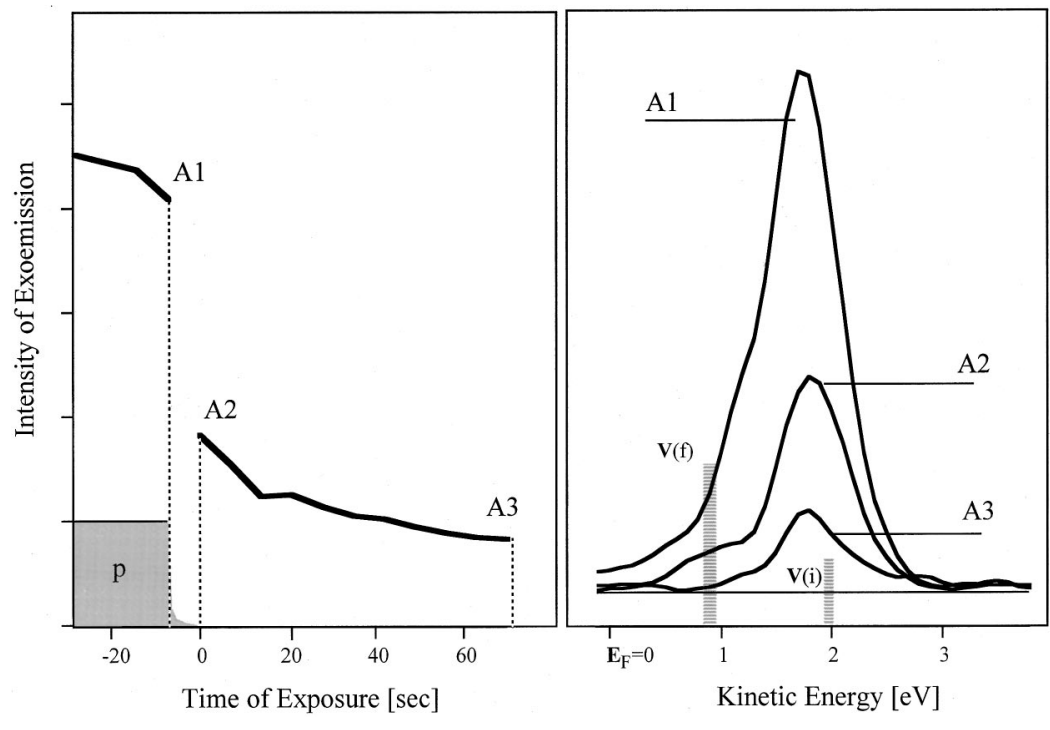

FIG. 3. The time evolution of the intensity of exoemission taken at the late oxidation stage, before and after shutting down of the $\mathrm{N}_{2} \mathrm{O}$ beam (left panel). The course of the total pressure in the chamber is shown only schematically. The panel on the right shows three energy distributions of the emitted electrons as monitored for three representative oxidation points $A 1, A 2$, and $A 3$. within the oxide-derived band gap, in the vicinity of the Fermi level, i.e., about $1 \mathrm{eV}$ below the vacuum level. Obviously, we might only tentatively attribute the origin of such a state to $\mathrm{O}$ vacancies, $V^{*}$, in the Cs-O network. Figure 4 illustrates the concept schematically. The steady-state occupation of the narrow state is warranted by ballistic electrons passing through the oxide layer. The lifetime of this kind of excitation is governed by two relaxation channels indicated by black arrows, I and II. Channel I becomes active when electrons escape the state $V^{*}$ and occupy the empty top states of the valence band. The relaxation path II becomes open when the empty LUMO state of the impinging $\mathrm{N}_{2} \mathrm{O}$ molecule dives deep below the $V^{*}$ state, close to the top of the valence band. The latter requirement is fulfilled nearly automatically, because the vertical affinity of nitrous oxides is up to $2.2 \mathrm{eV}$ (Ref. 31) which coincides roughly with the energy position of the highest $\mathrm{O}-2 p$ derived state of about $2.5 \mathrm{eV}$ below the vacuum level. ${ }^{32}$ The two electronic transi-

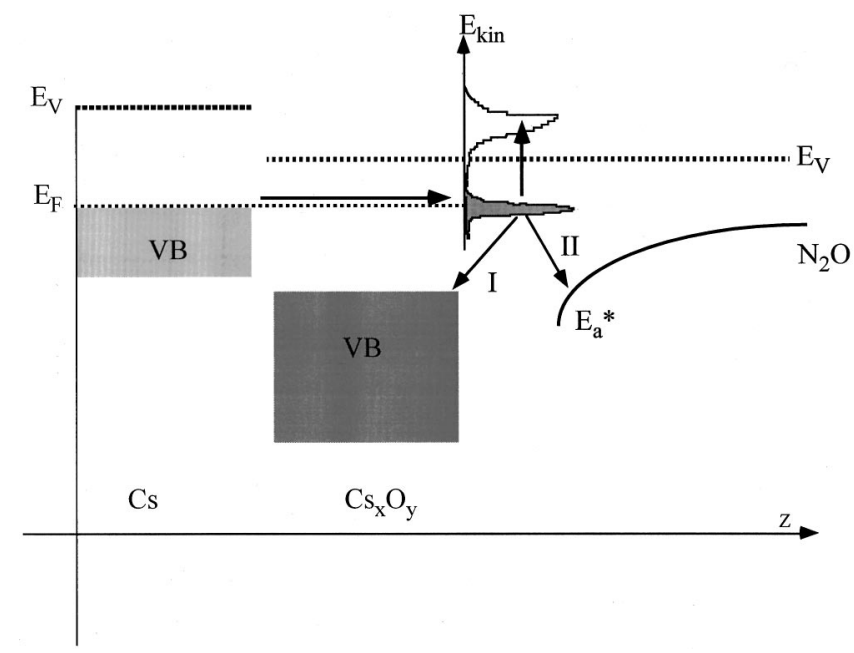

FIG. 4. A diagram showing the electronic situation created after oxidizing several top layers of the Cs film. The bold arrows show the two possible relaxation channels followed by the Auger deexcitation (thin arrow). tions generate quite similar Auger cascades, which finally lead to the energy distribution of exoemission as shown in Fig. 3. This way, both the chemisorptive as well as the postchemisorptive electron emission have a common origin that guarantees comparable energy distributions of emitted electrons. When combining the typical value of the probability for Auger de-excitation of $10^{-3}$, and the flux of impinging $\mathrm{N}_{2} \mathrm{O}$ molecules of about $10^{13} \mathrm{~cm}^{-2} \mathrm{~s}^{-1}$, the total flux of emitted electrons of $10^{10} \mathrm{~s}^{-1}$ can be derived for the oxidation stage marked by $A 1$ (Fig. 3). The fact that the emission observed within the postchemisorptive stage $(A 2-A 3)$ is in average five times less intense, implies that the lifetime of state $V^{*}$ lies in the range of $10^{-12} \mathrm{~s}$.

Next we prove experimentally some evidence for the postulated narrow, localized state, schematically shown in Fig. 4. We applied the de-excitation of metastable $\mathrm{He}^{*}$ atoms (MDS, e.g. Ref. 33) known as a most surface sensitive spectroscopy, suitable for monitoring the electronic structure of the outermost surface layer. The $\operatorname{He}^{*}\left(2{ }^{1} S\right)$ atoms deexciting in front of the surface, reveal the same regions that are penetrated by $\mathrm{N}_{2} \mathrm{O}$ molecules during the chemisorptive path. Figure 5(a) shows the valence-band MD spectra of a thick Cs film measured during the exposure to the $\mathrm{N}_{2} \mathrm{O}$ beam. The spectrum of a clean $\mathrm{Cs}$ surface (a) exhibits all characteristic features known from the literature. ${ }^{21,34}$ At highest kinetic energy, we find two peaks, $S$ and $T$, separated by about $1.2 \mathrm{eV}$. Both originate from the de-excitation of singlet and triplet metastable atoms on delocalized Cs- $6 s$ metallic states. A weak feature located $2.1 \mathrm{eV}$ below the triplet peak $(T)$ represents the surface-plasmon loss and the peak centered at $11 \mathrm{eV}$ represents the $\mathrm{O}_{3} \quad V V$-Auger de-excitation. ${ }^{33,34}$ The MD spectra of an oxidized Cs film ( $b$, $c$, and $d$ ) exhibit only two features located at kinetic energies of 14 and $18.2 \mathrm{eV}$, marked by $\mathrm{O}^{*}$ and $V^{*}$, respectively. Whereas the low-energy peak grows up, the high-energy peak decreases gradually with progressing $\mathrm{N}_{2} \mathrm{O}$ exposure. This energy position as well as the evident trend, allows us to assign peak $\mathrm{O}^{*}$ to a surface oxygen species. Peak $V^{*}$, however, can be associated with the vacancy state proposed 

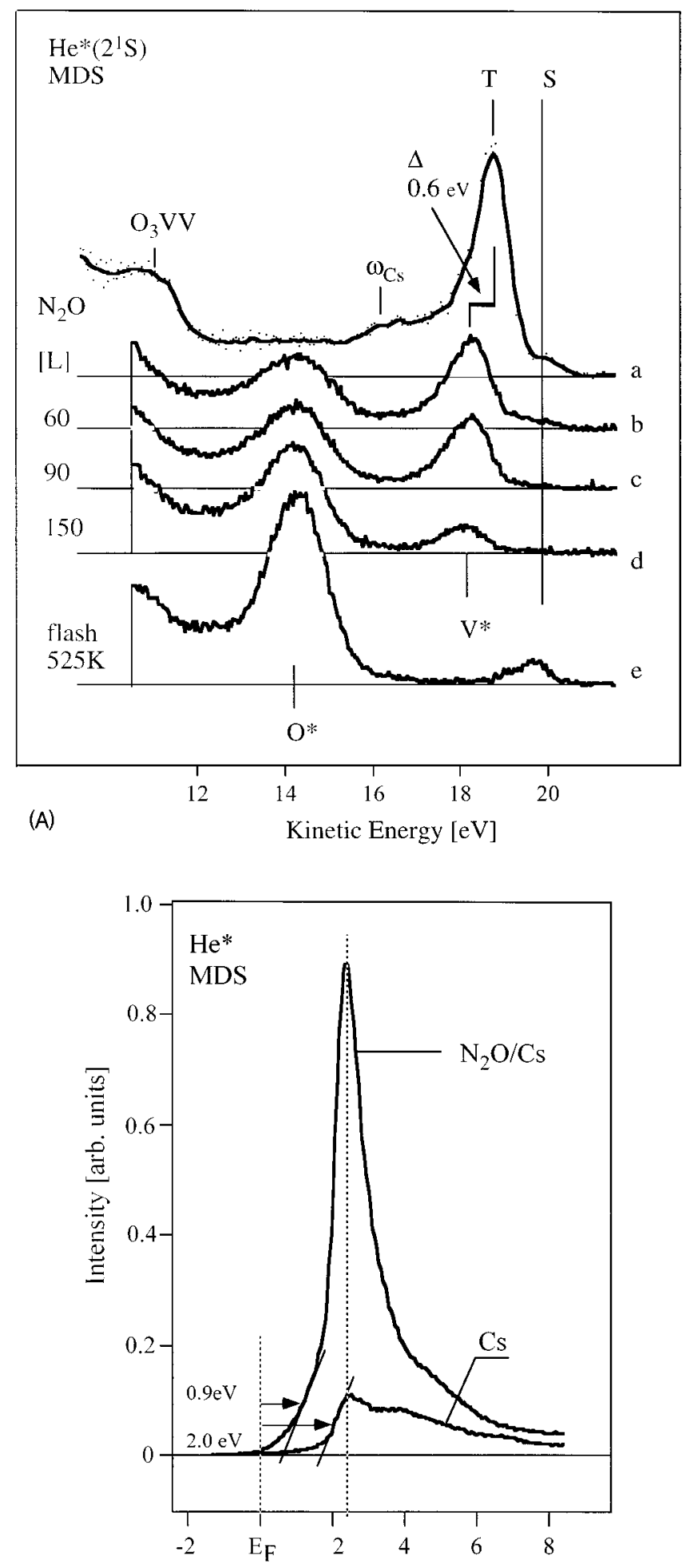

(B)

Kinetic Energy [eV]

FIG. 5. (A) shows the valence region of the $\mathrm{He}^{*}\left(2^{1} S\right)$ deexcitation spectra taken during the exposure of a thick Cs film to a constant $\mathrm{N}_{2} \mathrm{O}$ pressure of $5 \times 10^{-7}$ mbar [traces (a)-(d).] Spectrum (e) mirrors the situation after heating the sample up to $525 \mathrm{~K}$. Panel (B) shows the energy region of the MD spectra close to the vacuum level as monitored before and after exposing the thick Cs film to $150 \mathrm{~L}$ of $\mathrm{N}_{2} \mathrm{O}$, lower and upper spectrum, respectively. above, because it is located close to the Fermi level and becomes gradually quenched due to an increasing oxygen incorporation. Moreover, this state disappears completely when removing Cs bulk atoms by heating the sample up to $525 \mathrm{~K}$ (spectrum $e$ ). The surface rearranged this way represents a $2 \mathrm{D}$ system of $\mathrm{Cs}$ and $\mathrm{O}$ atoms coadsorbed on the $\mathrm{Ru}$ substrate. The corresponding spectra exhibit a very strong peak $\mathrm{O}^{*}$ and another weak feature at a kinetic energy of about $20 \mathrm{eV}$, i.e., the energy where the de-excitation of singlet $\mathrm{He}^{*}$ has been observed for a thick clean Cs film. According to the mechanism of the singlet-triplet conversion process, the $S$ peak has been attributed to the de-excitation of singlet $\mathrm{He}^{*}$ taking place on localized Cs- $6 s$ orbitals surrounded by coadsorbed oxygen atoms. ${ }^{21}$ The large energy separation between peaks $V^{*}$ and $S$ of $1.6 \mathrm{eV}$ does not directly reflect the real energy position of the $V^{*}$ state within the band gap. It reveals the quite different de-excitation scenario in the two cases. The extremely low work function (1 $\mathrm{eV}$ ) of the oxidized Cs film opens a new channel for surfacemolecule electron transfer. The affinity level of the impinging $\mathrm{He}^{*}\left(2{ }^{1} S\right)$, the so-called $\mathrm{He}^{-}\left(1 s 2 s^{2}{ }^{2} S\right)$ resonance, is placed slightly below state $V^{*}(1.25 \mathrm{eV})$ already at an infinite molecule-surface distance. Thus the impinging metastables become transformed into excited negative ions far from the surface. This fact assures an efficient singlet-triplet conversion to occur according to the mechanism proposed by Hemmen and Conrad $^{35}$ and theoretically described by Borisov, Teillet-Billy, and Gauyacq. ${ }^{36}$ Hence, feature $V^{*}$ placed $1.6 \mathrm{eV}$ below peak $S$ can be assigned to the deexcitation of triplet $\mathrm{He}^{*}\left(2^{3} S\right)$.

It has to be noted that state $V^{*}$ does not exist when performing the oxidation of Cs submonolayers. In this case the highest-energy peak resembles the one shown in spectrum $e$ in Fig. 5(A); thus it can be attributed to the de-excitation of singlet $\mathrm{He}^{*}$.

We found a second experimental support illustrating the role of state $V^{*}$. Figure 5(b) shows the low-energy region of two MD spectra taken from a clean and oxidized thick Cs film. The latter exhibits a giant emissive feature with a maximum around $2.2 \mathrm{eV}$ above $E_{F}$. This energy position overlaps with the energy distribution of exoelectrons emitted within the $A 2-A 3$ interval (Fig. 3) which suggests a common mechanism for the two phenomena. Namely, the abovementioned Auger de-excitation, initialized by the relaxation of state $V^{*}$ (as shown in Fig. 4). Impinging $\mathrm{He}^{*}$ atoms induce various primary as well as secondary electronic processes. $^{37}$ The latter represents a second source for electrons, which in addition to the ballistic electrons populate state $V^{*}$ very efficiently and consequently generate the intense peak above the vacuum level.

\section{SUMMARY}

By comparing the time evolution of the sticking probability with the kinetics of the electron emission monitored during the $\mathrm{N}_{2} \mathrm{O}$ chemisorption on Cs films, two oxidation stages can be distinguished. Within the initial stage, the interaction of impinging $\mathrm{N}_{2} \mathrm{O}$ with the still metallic Cs film is governed mainly by the long-range surface harpooning, which is re- 
sponsible for the similarity in the behavior of sticking and the electron emission. The late oxidation stage starts after the work function reaches a minimum of about $1 \mathrm{eV}$, which signalizes the formation of a saturated layer of surface suboxides. Within the late oxidation stage the decaying sticking probability is accompanied by a pronounced increase of the emission intensity. This late-stage electron emission is continued even when interrupting the $\mathrm{N}_{2} \mathrm{O}$ chemisorption. The late-stage chemisorptive exoemission reflects the Auger cascade induced by an empty affinity state appearing at the top of the valence band when the $\mathrm{N}_{2} \mathrm{O}$ molecule sticks to the surface. The nonchemisorptive emission mirrors an internal electron transition from the Fermi region into some unoccupied states on top of the valence band. Both channels become intelligible when considering a common oxygen-vacancy de- rived state located inside the band gap as the mediator for the induced Auger de-excitation. The required considerable steady-state occupation of this state is warranted by ballistic electrons stemming from the still metallic Cs layer in beneath. The MD spectra of thick oxidized Cs films reveal an additional peak that fulfills all requirements of an vacancy derived state.

\section{ACKNOWLEDGMENTS}

This work was partly supported by the Deutsche Forschungsgemeinschaft (Ni-452) during building a new molecular-beam apparatus at the Humboldt-University. A. B. thanks the directorate of the Fritz-Haber-Institut for financial support enabling a successful completion of this work.
*Author to whom correspondence should be addressed: Email address: boettche@fhi-berlin.mpg.de FAX: 049-30-8413-5603.

${ }^{1}$ J. K. Nørskov, D. M. Newns, and B. I. Lundqvist, Surf. Sci. 80, 179 (1979).

${ }^{2}$ A. Böttcher, R. Imbeck, A. Morgante, and G. Ertl, Phys. Rev. Lett. 65, 2035 (1991).

${ }^{3}$ A. Böttcher, R. Grobecker, R. Imbeck, A. Morgante, and G. Ertl, J. Chem. Phys. 95, 3756 (1991).

${ }^{4}$ T. Greber, Surf. Sci. Rep. 28, 1 (1997).

${ }^{5}$ A. Böttcher, A. Morgante, and G. Ertl, Surf. Sci. Lett. 359, 461 (1996).

${ }^{6}$ A. Böttcher and T. Gießel, Surf. Sci. 408, 212 (1998).

${ }^{7}$ R. Grobecker, H. Shi, H. Bludau, T. Hertel, T. Greber, A. Böttcher, K. Jacobi, and G. Ertl, Phys. Rev. Lett. 72, 578 (1994).

${ }^{8}$ T. Greber, Chem. Phys. Lett. 222, 292 (1994).

${ }^{9}$ K. Hermann, K. Freihube, T. Greber, A. Böttcher, R. Grobecker, D. Fick, and G. Ertl, Surf. Sci. Lett. 313, 806 (1994).

${ }^{10}$ A. Böttcher and H. Niehus, Proceedings of the International Symposium on Exoemission and its Applications (Glucholazy, 1994), p. 133.

${ }^{11}$ T. Greber, K. Freihube, R. Grobecker, A. Böttcher, K. Hermann, G. Ertl, and D. Fick, Phys. Rev. B 50, 8755 (1994).

${ }^{12}$ M. P. Cox, J. S. Foord, R. M. Lambert, and R. H. Prince, Surf. Sci. 129, 399 (1983).

${ }^{13}$ A. Böttcher, R. Grobecker, T. Greber, and G. Ertl, Chem. Phys. Lett. 208, 404 (1993).

${ }^{14}$ A. Böttcher, S. Fichnter-Endruschat, M. Wohlers, and H. Niehus, Surf. Sci. 388, 177 (1997).

${ }^{15}$ A. Böttcher, A. Morgante, T. Gießel, T. Greber, and G. Ertl, Chem. Phys. Lett. 231, 119 (1994).

${ }^{16}$ L. Hellberg, J. Strömqvist, B. Kasemo, and B. I. Lundtqvist, Phys. Rev. Lett. 74, 4742 (1995).

${ }^{17}$ M. Brandt, T. Greber, N. Böwering, and U. Heinzmann, Phys. Rev. Lett. 81, 2376 (1998).
${ }^{18}$ M. Brandt, T. Greber, F. Kuhlmann, N. Böwering, and U. Heinzmann, Surf. Sci. 160, 402 (1998).

${ }^{19}$ H. Over, B. Bludau, M. Skottke-Klein, G. Ertl, W. Moritz, and C. T. Campbell, Phys. Rev. B 45, 8638 (1992).

${ }^{20}$ H. Bludau, H. Over, T. Hertel, M. Gierer, and G. Ertl, Surf. Sci. 342, 134 (1995).

${ }^{21}$ A. Böttcher, A. Morgante, R. Grobecker, T. Greber, and G. Ertl, Phys. Rev. B 49, 10607 (1994).

${ }^{22}$ Y. Li and J. N. Armor, Appl. Catl. B 1, 21 (1992).

${ }^{23}$ K. Yuzaki, T. Yarimizu, K. Aoyagi, S-I. Ito, T. Sato, S. Hayashi, and K. Kunimori, Catal. Today 45, 129 (1998).

${ }^{24}$ T. E. Madey, T. Engelhardt, and D. Menzel, Surf. Sci. 48, 304 (1975).

${ }^{25}$ D. A. King and M. G. Wells, Proc. R. Soc. London, Ser. A 339, 245 (1974).

${ }^{26}$ S. K. Shi, H. I. Lee, and J. M. White, Surf. Sci. 102, 56 (1981).

${ }^{27}$ P. Kisliuk, J. Phys. Chem. Solids 5, 78 (1958).

${ }^{28}$ M. Kiskinova, G. Rangelow, and L. Surnev, Surf. Sci. 172, 57 (1986).

${ }^{29}$ E. V. Albano, Surf. Sci. 141, 191 (1984).

${ }^{30}$ C. Y. Su, I. Lindau, P. W. Chye, S. J. Oh, and W. E. Spicer, J. Electron Spectrosc. 31, 221 (1983).

${ }^{31}$ D. G. Hopper, A. C. Wahl, R. L. C. Wu, and T. O. Tiernan, J. Chem. Phys. 65, 5474 (1976).

${ }^{32}$ G. Ebbinghaus and A. Simon, Chem. Phys. 43, 117 (1970).

${ }^{33}$ G. H. Rocker, C. L. Cobb, H.-S. Jun, H. Metiu, and R. M. Martin, Surf. Sci. 208, 20 (1989).

${ }^{34}$ B. Woratschek, W. Sesselmann, J. Küppers, and G. Ertl, J. Chem. Phys. 86, 2411 (1987).

${ }^{35}$ R. Hemmen and H. Conrad, Phys. Rev. Lett. 67, 1314 (1991).

${ }^{36}$ A. G. Borisov, D. Teillet-Billy, and J. P. Gauyacq, Surf. Sci. 284, 337 (1993).

${ }^{37}$ A. Böttcher, Langmuir 16, 8858 (2000). 